PROCEEDINGS OF THE

AMERICAN MATHEMATICAL SOCIETY

Volume 128, Number 5, Pages 1481-1486

S 0002-9939(99)05404-0

Article electronically published on October 5, 1999

\title{
TIGHTNESS BOUNDS FOR STRONGLY MIXING RANDOM SEQUENCES
}

\author{
RICHARD C. BRADLEY
}

(Communicated by Stanley Sawyer)

\begin{abstract}
For a given strictly stationary, strongly mixing random sequence for which the distributions of the partial sums are tight, certain "tightness bounds" exist which depend only on the marginal distribution and the mixing rate.
\end{abstract}

\section{INTRODUCTION}

Suppose $X:=\left(X_{k}, k \in \mathbb{Z}\right)$ is a strictly stationary sequence of (real-valued) random variables on a probability space $(\Omega, \mathcal{F}, P)$. For $-\infty \leq J \leq L \leq \infty$, let $\mathcal{F}_{J}^{L}$ denote the $\sigma$-field generated by the random variables $X_{k}, J \leq k \leq L(k \in \mathbb{Z})$. For $\sigma$-fields $\mathcal{A}$ and $\mathcal{B}$, define the measure of dependence

$$
\alpha(\mathcal{A}, \mathcal{B}):=\sup _{A \in \mathcal{A}, B \in \mathcal{B}}|P(A \cap B)-P(A) P(B)| .
$$

For each $n=1,2,3, \ldots$, define the dependence coefficient

$$
\alpha(n)=\alpha(X, n):=\alpha\left(\mathcal{F}_{-\infty}^{0}, \mathcal{F}_{n}^{\infty}\right) .
$$

The (strictly stationary) sequence $X$ is "strongly mixing" 8 if $\alpha(n) \rightarrow 0$ as $n \rightarrow \infty$.

For the given (strictly stationary) sequence $X$, define for each $n \geq 1$ the partial sum $S_{n}:=X_{1}+X_{2}+\cdots+X_{n}$.

If $V$ and $W$ are nonnegative random variables, we shall say that $V$ is "stochastically $\leq W$ " if $P(V>t) \leq P(W>t)$ for all $t \geq 0$. Of course, if $V \leq W$ a.s., then $V$ is stochastically $\leq W$.

The probability space is of course assumed to be purely nonatomic, so that random variables and random sequences with any distribution can be defined on that space.

Here is the main result of this note:

Theorem 1.1. Suppose $V$ is a nonnegative random variable, and $q:=\left(q_{1}, q_{2}, q_{3}, \ldots\right)$ is a nonincreasing sequence of nonnegative numbers such that $q_{n} \rightarrow 0$ as $n \rightarrow \infty$. Then there exists a nonnegative random variable $W$ such that the following holds:

Received by the editors June 25, 1998.

2000 Mathematics Subject Classification. Primary 60G10; Secondary 60G07.

Key words and phrases. Strictly stationary, strong mixing, tightness.

This work was partially supported by NSF grant DMS 9703712. 
Suppose $X:=\left(X_{k}, k \in \mathbb{Z}\right)$ is a strictly stationary sequence of random variables such that

(i) the random variable $\left|X_{0}\right|$ is stochastically $\leq V$,

(ii) $\alpha(X, n) \leq q_{n}$ for all $n \geq 1$, and

(iii) the family of distributions of the partial sums $\left(S_{n}, n \geq 1\right)$ is tight.

Then for each $n \geq 1$, the random variable $\left|S_{n}\right|$ is stochastically $\leq W$.

This theorem will be proved in section 2 . For strictly stationary, strongly mixing random sequences whose partial sums satisfy tightness, it gives in essence a tightness "bound" that depends only on the marginal distribution and the mixing rate. The random variable $W$ depends on $V$ and on the sequence $q$; that dependence is somewhat complicated, but can be seen from the proof.

As a corollary, using "symmetrization" as in the proof of [1 Theorem 2], one can derive an analog of Theorem 1 with "tightness" replaced by "shift tightness" (that is, with the family of distributions of $S_{n}-m_{n}, n \geq 1$, being tight, where $m_{n}$ denotes a median of $S_{n}$ ). The details will not be given here.

Now let us consider the special case of bounded random variables.

Theorem 1.2. Suppose $\theta>0$. Suppose $X:=\left(X_{k}, k \in \mathbb{Z}\right)$ is a strictly stationary sequence of bounded random variables such that $\alpha(X, n)=O\left(n^{-\theta}\right)$ as $n \rightarrow \infty$, and the family of distributions of the partial sums $\left(S_{n}, n \geq 1\right)$ is tight. Then for every $p \in(0, \theta)$, one has that $\sup _{n \geq 1} E\left|S_{n}\right|^{p}<\infty$.

Remark 1.3. Theorem 1.2 will be proved in section 2 . Some motivation and variations will be given in the following comments, and also in Remark 2.3 at the end of section 2 .

(a) Suppose $\left(Z_{k}, k \in \mathbb{Z}\right)$ is a sequence of independent, identically distributed random variables such that $P\left(Z_{0}=1\right)=P\left(Z_{0}=-1\right)=1 / 2$. Suppose the random sequence $Y:=\left(Y_{k}, k \in \mathbb{Z}\right)$ is defined by $Y_{k}=Z_{k}-Z_{k-1}, k \in \mathbb{Z}$. This sequence $Y$ is strictly stationary and strongly mixing (in fact 1-dependent). For each $n \geq$ $1, Y_{1}+\cdots+Y_{n}=Z_{n}-Z_{0}$ (from a telescoping sum), and therefore $P\left(Y_{1}+\cdots+Y_{n}=\right.$ $-2,0$, or 2$)=1$. In this classic example, the partial sums do not "grow" (in probability), and do not satisfy (say) a central limit theorem.

(b) In central limit theorems for strictly stationary, strongly mixing sequences $\left(X_{k}, k \in \mathbb{Z}\right)$ of (not necessarily bounded) random variables with mean 0 and finite second moments, an assumption that $E S_{n}^{2} \rightarrow \infty$ as $n \rightarrow \infty$ is often used (in conjunction with other assumptions) in order to insure that $S_{n}$ "grows" (in probability) and examples such as the one in (a) above are avoided. See for example the central limit theorems in [4, 5], 7, 8].

(c) However, Herrndorf [6 constructed a strictly stationary, strongly mixing sequence $\left(X_{k}, k \in \mathbb{Z}\right)$ of (unbounded) random variables with mean 0 and finite second moments, with $E S_{n}^{2} \rightarrow \infty$ as $n \rightarrow \infty$, such that the family of distributions of the partial sums $\left(S_{n}, n \geq 1\right)$ is tight (and $S_{n}$ fails to satisfy a central limit theorem). In that example, the mixing rate can be taken arbitrarily fast.

(d) Now suppose $\left(X_{k}, k \in \mathbb{Z}\right)$ is a strictly stationary, strongly mixing sequence of bounded random variables with mean 0 . If $E S_{n}^{2} \rightarrow \infty$ and $\sum_{n=1}^{\infty} n \cdot \alpha(n)<\infty$, then $S_{n}$ "grows" (in probability) and satisfies a central limit theorem. This has long been known; it can be seen e.g. as a special case of [3, p. 511, Corollary 1, and p. 528, lines 18 and 19]. If $E S_{n}^{2} \rightarrow \infty$ but the mixing rate is only slightly slower, for example $\alpha(n)=O\left(n^{-2}\right)$ as $n \rightarrow \infty$, then the family of distributions of 
the partial sums $\left(S_{n}, n \geq 1\right)$ can be tight, and $S_{n}$ can fail to satisfy a central limit theorem. This can be seen from [3, p. 527, Theorem 2, and p. 528, Example 1]. The construction there incorporated some ideas from the construction of Herrndorf [6] discussed in (c) above.

(e) Comment (d) shows that, at least in the case $\theta=2$, Theorem 1.2 is quite sharp: In Theorem 1.2, if $\alpha(n)=O\left(n^{-2}\right)$, then $\sup _{n>1} E\left|S_{n}\right|^{p}<\infty$ for all $p \in(0,2)$, but $\sup _{n \geq 1} E S_{n}^{2}$ may be $\infty$.

(f) For a given $\theta>0$, Theorem 1.2 can be strengthened slightly in different ways, with just trivial modifications of the proof. For example, the mixing rate can be marginally slower. Also, one can construct a nonnegative random variable $W$ with $E W^{p}<\infty \forall p \in(0, \theta)$, such that, for all $n \geq 1,\left|S_{n}\right|$ is stochastically $\leq W$.

(g) With a similar proof, one can show that if $\left(X_{k}, k \in \mathbb{Z}\right)$ is a strictly stationary, $m$-dependent sequence of bounded random variables and the family of distributions of $S_{n}, n \geq 1$, is tight, then $\sup _{n \geq 1}\left\|S_{n}\right\|_{\infty}<\infty$.

\section{Proofs}

First we need some notation. For events $A$ and $B$, the notation $A \doteq B$ will mean that $P(A \triangle B)=0$, where $\triangle$ denotes symmetric difference. If $\mathcal{A}$ and $\mathcal{B}$ are $\sigma$-fields, the notation $\mathcal{A} \subset \mathcal{B}$ will mean that $\forall A \in \mathcal{A}, \exists B \in \mathcal{B}$ such that $A \doteq B$; and the notation $\mathcal{A} \doteq \mathcal{B}$ will mean that $\mathcal{A} \dot{\subset} \mathcal{B}$ and $\mathcal{B} \dot{\subset} \mathcal{A}$.

The $\sigma$-field generated by a family $\left(\zeta_{i}, i \in I\right)$ of random variables (where $I$ is an index set) will be denoted $\sigma\left(\zeta_{i}, i \in I\right)$.

For a given random variable $\zeta$, the least median will be denoted $\operatorname{lmed}(\zeta)$. This is the least real number $r$ such that $P(\zeta \leq r) \geq 1 / 2$.

Lemma 2.1. Suppose $Y:=\left(Y_{k}, k \in \mathbb{Z}\right)$ is a strictly stationary, ergodic sequence of random variables, and the family of distributions of its partial sums $\left(Y_{1}+Y_{2}+\right.$ $\left.\cdots+Y_{n}, n=1,2,3, \ldots\right)$ is tight. Then there exists a strictly stationary, ergodic sequence $Z:=\left(Z_{k}, k \in \mathbb{Z}\right)$ of random variables with the following four properties:

(a) $\forall k \in \mathbb{Z}, Y_{k}=Z_{k}-Z_{k-1}$ a.s.

(b) $\forall k \in \mathbb{Z}, \sigma\left(Z_{k}\right) \dot{\subset} \sigma\left(Y_{k+1}, Y_{k+2}, Y_{k+3}, \ldots\right)$.

(c) $\forall k \in \mathbb{Z}, \sigma\left(Z_{k}\right) \dot{\subset} \sigma\left(Y_{k}, Y_{k-1}, Y_{k-2}, \ldots\right)$.

(d) $\forall n \geq 1, \alpha(Z, n)=\alpha(Y, n+1)$.

In particular, in the context of Lemma 2.1, if $Y$ is strongly mixing, then $Z$ is strongly mixing. (The same applies to various other mixing conditions, such as absolute regularity, $\rho$-mixing, $\phi$-mixing, or $\psi$-mixing.)

Lemma 2.1 (without (d)) is taken from Schmidt 9, Lemma 11.7]. The formulation and proof are given there in "ergodic theoretic" terminology. For readers more accustomed to "probability theoretic" terminology, a more convenient formulation and proof (a modification of Schmidt's argument) are given in [2, Theorem 1 and p. 9, line -10]. The formulation there includes both (a) and (b). Subtracting a constant from the $Z_{k}$ 's if necessary, one can assume without loss of generality that $\operatorname{lmed}\left(Z_{0}\right)=0$. Then (c) follows from (a) and ergodicity by a standard argument (used e.g. in [9]); that argument is spelled out (for $k=0$ ) in the same terminology in [1, p. 289, lines 1 to 16]. (Similarly, (b) is also a consequence of (a) and ergodicity.) Now only (d) remains to be verified.

Suppose that $n \geq 1$. One has $\sigma\left(Y_{k}, k \geq n+1\right) \dot{\subset} \sigma\left(Z_{k}, k \geq n\right)$ by (a), and $\sigma\left(Z_{k}, k \geq n\right) \dot{\subset} \sigma\left(Y_{k}, k \geq n+1\right)$ by (b). Hence $\sigma\left(Y_{k}, k \geq n+1\right) \doteq \sigma\left(Z_{k}, k \geq n\right)$. Similarly by (a) and (c), $\sigma\left(Y_{k}, k \leq 0\right) \doteq \sigma\left(Z_{k}, k \leq 0\right)$. Property (d) follows. 
Lemma 2.2. Suppose $Y:=\left(Y_{k}, k \in \mathbb{Z}\right)$ is a strictly stationary, ergodic sequence of random variables, and the family of distributions of its partial sums $\left(Y_{1}+\cdots+\right.$ $\left.Y_{n}, n \geq 1\right)$ is tight. Suppose $c$ and $\delta$ are nonnegative numbers such that

$$
P\left(\left|Y_{0}\right|>c\right) \leq \delta
$$

Then for any positive integers $m$ and $n$, one has that

$$
P\left(\left|Y_{1}+\cdots+Y_{n}\right|>2 m c\right) \leq 8 \cdot[m \delta+\alpha(Y, m+1)] .
$$

Proof. Let $m$ and $n$ each be an arbitrary, fixed positive integer. Our task is to verify $(2.2)$.

Let $Z:=\left(Z_{k}, k \in \mathbb{Z}\right)$ be a strictly stationary, ergodic sequence as in Lemma 2.1, satisfying properties (a), (b), (c), and (d) there. Without loss of generality, assume that

$$
\operatorname{lmed}\left(Z_{0}\right)=0 .
$$

In order to verify $(2.2)$, we first need to work with the sum $Y_{1}+\cdots+Y_{m}$ (instead of $\left.Y_{1}+\cdots+Y_{n}\right)$.

By (2.1), stationarity, and property (a) in Lemma 2.1, one has that

$$
P\left(Z_{m}-Z_{0}>m c\right)=P\left(Y_{1}+\cdots+Y_{m}>m c\right) \leq m \cdot P\left(Y_{0}>c\right) \leq m \delta .
$$

Hence by (2.3), stationarity, and property (d) in Lemma 2.1,

$$
\begin{aligned}
m \delta & \geq P\left(Z_{m}-Z_{0}>m c\right) \geq P\left(Z_{m}>m c \text { and } Z_{0} \leq 0\right) \\
& \geq P\left(Z_{m}>m c\right) \cdot P\left(Z_{0} \leq 0\right)-\alpha(Z, m) \\
& \geq P\left(Z_{m}>m c\right) \cdot(1 / 2)-\alpha(Z, m) \\
& =(1 / 2) \cdot P\left(Z_{0}>m c\right)-\alpha(Y, m+1)
\end{aligned}
$$

and hence

$$
P\left(Z_{0}>m c\right) \leq 2 \cdot[m \delta+\alpha(Y, m+1)] .
$$

By arguments analogous to those of (2.4) and (2.5), one has $P\left(Z_{m}-Z_{0}<-m c\right)$ $\leq m \delta$ and hence

$$
\begin{aligned}
m \delta & \geq P\left(Z_{m}<-m c \text { and } Z_{0} \geq 0\right) \\
& \geq(1 / 2) \cdot P\left(Z_{0}<-m c\right)-\alpha(Y, m+1),
\end{aligned}
$$

and hence $P\left(Z_{0}<-m c\right) \leq 2 \cdot[m \delta+\alpha(Y, m+1)]$. Combining this last inequality with (2.6), one has that

$$
P\left(\left|Z_{0}\right|>m c\right) \leq 4 \cdot[m \delta+\alpha(Y, m+1)] .
$$

Now we are ready to deal with the partial sum $Y_{1}+\cdots+Y_{n}$. By $(2.7)$, stationarity, and property (a) in Lemma 2.1, one has that

$$
\begin{array}{r}
P\left(\left|Y_{1}+\cdots+Y_{n}\right|>2 m c\right)=P\left(\left|Z_{n}-Z_{0}\right|>2 m c\right) \\
\leq 2 \cdot P\left(\left|Z_{0}\right|>m c\right) \leq 8 \cdot[m \delta+\alpha(Y, m+1)] .
\end{array}
$$

Thus (2.2) holds. This completes the proof of Lemma 2.2 .

Proof of Theorem 1.1. Let the nonnegative random variable $V$ and the sequence $q$ be as in the statement of Theorem 1.1.

Let $m_{1}, m_{2}, m_{3}, \ldots$ be a sequence of positive integers such that

$$
\forall j \geq 1, \quad m_{j+1}>m_{j} \quad \text { and } \quad q_{m(j)+1} \leq 2^{-j-4} .
$$


(Here $m(j)$ means $m_{j}$.) Let $\delta_{1}, \delta_{2}, \delta_{3}, \ldots$ be a sequence of positive numbers such that

$$
\forall j \geq 1, \quad 8 m_{j} \delta_{j} \leq 2^{-j-1} .
$$

Let $c_{1}, c_{2}, c_{3}, \ldots$ be a sequence of positive numbers such that

$$
\forall j \geq 1, \quad c_{j+1}>c_{j} \text { and } P\left(V>c_{j}\right) \leq \delta_{j} .
$$

For each $j \geq 1$, define the positive number

$$
A_{j}:=2 m_{j} c_{j} .
$$

By (2.8) and (2.10) (and the fact that the $m_{j}$ 's are integers),

$$
A_{1}<A_{2}<A_{3}<\ldots, \text { and } A_{j} \rightarrow \infty \text { as } j \rightarrow \infty \text {. }
$$

Let $W$ be a (positive discrete) random variable taking the values $A_{1}, A_{2}, A_{3}, \ldots$, such that

$$
\forall j \geq 1, P\left(W=A_{j}\right)=2^{-j} .
$$

Now suppose $X:=\left(X_{k}, k \in \mathbb{Z}\right)$ is a strictly stationary random sequence as in Theorem 1.1, satisfying properties (i), (ii), and (iii) there. Let $n \geq 1$ and $t \geq 0$ be arbitrary but fixed. In order to complete the proof of Theorem 1.1, it suffices to show that

$$
P\left(\left|S_{n}\right|>t\right) \leq P(W>t) .
$$

Refer to (2.12) and (2.13). If $0 \leq t<A_{1}$, then $1=P\left(W \geq A_{1}\right)=P(W>t)$, and hence (2.14) holds and we are done. Therefore, let us assume instead that $t \geq A_{1}$.

Referring to (2.12), let $j$ be the positive integer such that

$$
A_{j} \leq t<A_{j+1} .
$$

By (2.10) and condition (i) in Theorem 1.1, one has $P\left(\left|X_{0}\right|>c_{j}\right) \leq P\left(V>c_{j}\right)$ $\leq \delta_{j}$. Hence by (2.15), (2.11), Lemma 2.2, and conditions (ii) and (iii) in Theorem 1.1 , followed by (2.8) and (2.9), one has that

$$
\begin{aligned}
P\left(\left|S_{n}\right|>t\right) & \leq P\left(\left|S_{n}\right|>A_{j}\right)=P\left(\left|S_{n}\right|>2 m_{j} c_{j}\right) \\
& \leq 8\left[m_{j} \delta_{j}+\alpha\left(X, m_{j}+1\right)\right] \\
& \leq 8 m_{j} \delta_{j}+8 q_{m(j)+1} \leq 2^{-j} .
\end{aligned}
$$

Also by (2.12), (2.13), and (2.15),

$$
P(W>t)=P\left(W \geq A_{j+1}\right)=\sum_{\ell=j+1}^{\infty} 2^{-\ell}=2^{-j} .
$$

Hence by (2.16), eq. (2.14) holds. This completes the proof of Theorem 1.1.

Proof of Theorem 1.2. Suppose $\theta>0$. Suppose $X:=\left(X_{k}, k \in \mathbb{Z}\right)$ is as in the hypothesis of Theorem 1.2. Multiplying the $X_{k}$ 's by a positive constant if necessary, we assume without loss of generality that $\left|X_{0}\right| \leq 1$ a.s. Let $Q>0$ be such that $\alpha(X, n) \leq Q n^{-\theta}$ for all $n \geq 1$. Then by Lemma 2.2 (with $c=1$ and $\delta=0$ ), one has that, for any positive integers $m$ and $n$,

$$
P\left(\left|S_{n}\right|>2 m\right) \leq 8 \alpha(X, m+1) \leq 8 Q(m+1)^{-\theta} .
$$


Hence for any positive integer $n$, any $p \in(0, \theta)$, and any $t \geq 2^{p}$, letting $m$ denote the greatest (positive) integer $\leq t^{1 / p} / 2$, one has that

$$
\begin{aligned}
P\left(\left|S_{n}\right|>t^{1 / p}\right) & \leq P\left(\left|S_{n}\right|>2 m\right) \\
& \leq 8 Q(m+1)^{-\theta}<8 Q\left(t^{1 / p} / 2\right)^{-\theta} .
\end{aligned}
$$

Hence for any positive integer $n$ and any $p \in(0, \theta)$, letting $\Lambda:=2^{p}$, one has that

$$
\begin{aligned}
E\left|S_{n}\right|^{p} & =\int_{0}^{\infty} P\left(\left|S_{n}\right|^{p}>t\right) d t \\
& \leq \int_{0}^{\Lambda} 1 d t+\int_{\Lambda}^{\infty} P\left(\left|S_{n}\right|>t^{1 / p}\right) d t \\
& \leq 2^{p}+\int_{\Lambda}^{\infty} 2^{\theta} \cdot 8 Q \cdot t^{-\theta / p} d t .
\end{aligned}
$$

Since $\theta / p>1$ (if $p \in(0, \theta)$ ), the last term is finite, and also it doesn't depend on $n$. Theorem 1.2 follows.

Remark 2.3. Suppose $\left(X_{k}, k \in \mathbb{Z}\right)$ is as in Theorem 1.1 or Theorem 1.2, and (see Lemma 2.1) $\left(Z_{k}, k \in \mathbb{Z}\right)$ is a strictly stationary, ergodic sequence such that $\forall k \in \mathbb{Z}$, $X_{k}=Z_{k}-Z_{k-1}$ a.s. (Then all other properties in Lemma 2.1 hold as well; see the comments after Lemma 2.1.) From the proofs of Theorems 1.1 and 1.2 (including the proof of Lemma 2.2), one can see the following: In the context of Theorem $1.1,\left|Z_{0}-\operatorname{lmed}\left(Z_{0}\right)\right|$ is stochastically $\leq W$; and in the context of Theorem 1.2, $E\left|Z_{0}\right|^{p}<\infty$ for all $p \in(0, \theta)$. Similar analogs of Remark 1.3(f)(g) hold as well.

\section{REFERENCES}

[1] R.C. Bradley, On the dissipation of partial sums from a stationary strongly mixing sequence, Stochastic Process. Appl. 54 (1994), 281-290. MR 95j:60054

[2] R.C. Bradley, On a theorem of K. Schmidt, Statist. Probab. Letters 24 (1995), 9-12. MR 96g:60048

[3] R.C. Bradley, On quantiles and the central limit question for strongly mixing sequences, J. Theor. Probab. 10 (1997), 507-555. MR 98h:60024

[4] H. Dehling, M. Denker, and W. Philipp, Central limit theorems for mixing sequences of random variables under minimal conditions, Ann. Probab. 14 (1986), 1359-1370. MR 88d:60065

[5] M. Denker, Uniform integrability and the central limit theorem for strongly mixing processes, Dependence in Probability and Statistics (E. Eberlein, M.S. Taqqu, eds.), pp. 269-274, Birkhäuser, Boston, 1986. MR 88h:60044

[6] N. Herrndorf, Stationary strongly mixing sequences not satisfying the central limit theorem, Ann. Probab. 11 (1983), 809-813. MR 84m:60030

[7] T. Mori and K. Yoshihara, A note on the central limit theorem for stationary strong-mixing sequences, Yokohama Math J. 34 (1986), 143-146. MR 88g:60068

[8] M. Rosenblatt, A central limit theorem and a strong mixing condition, Proc. Natl. Acad. Sci. U.S.A. 42 (1956), 43-47. MR 17:635b

[9] K. Schmidt, Cocyles on Ergodic Transformation Groups, Macmillan, Delhi, 1977. MR 58:28262

Department of Mathematics, Indiana University, Bloomington, Indiana 47405-5701

E-mail address: bradleyr@indiana.edu 